\title{
A FUNÇÃO DE PRODUÇÃO DA INDÚSTRIA BRASILEIRA DE CELULOSE, EM 2004 ${ }^{1}$
}

\author{
Naisy Silva Soares², Márcio Lopes da Silva³ e João Eustáquio de Lima ${ }^{4}$
}

\begin{abstract}
RESUMO - O segmento de celulose e papel tem-se destacado como o mais importante do setor florestal no Brasil, e o conhecimento das relações de produção e das características da tecnologia pode gerar informações úteis para o setor. Este trabalho teve como objetivos especificar e ajustar a função de produção da indústria de celulose no Brasil, no ano de 2004, utilizando-se como referência os fundamentos da teoria da produção. Foi empregado um modelo Cobb-Douglas, em que as variáveis explicativas foram terra, trabalho e capital. As estimativas confirmaram que essas variáveis afetam, significativamente, a produção de celulose. Os resultados, usando o método dos Mínimos Quadrados Ordinários (MQO), apontaram que os fatores de produção analisados estão sendo empregados racionalmente na indústria de celulose no Brasil. Essa indústria apresenta retorno constantes à escala, terra e trabalho constituem nos fatores mais importantes na formação da quantidade produzida de celulose e as empresas mostram-se intensivas em capital.
\end{abstract}

Palavras-chave: Função de produção, celulose e setor florestal.

\section{THE PRODUCTION FUNCTION OF THE BRAZILIAN WOOD PULP INDUSTRY, IN 2004}

\begin{abstract}
The wood pulp and paper sector has become one of the most important segments of the forest industry in Brazil. Knowledge on production relationships and the technology characteristics can bring in important information for the sector. The main objective of the present work was to specify and fit the production function of wood pulp for the Brazilian industry, in 2004, on the basis of the production theory and using a Cobb-Douglas model with land, labor and capital as explanatory variables. The estimates showed that the explanatory variables significantly affected the wood pulp production. The results, using least squares method (LQO), indicated that the production factors are being rationally used in the Brazilian wood pulp industry; the pulp industry showed constant returns to scale; land and labor make up the most important factors in the formation of wood pulp production; and the companies are capital intensive.
\end{abstract}

Keywords: Production function, wood pulp and forest sector.

\section{INTRODUÇÃO}

O segmento de celulose e papel no Brasil é um dos mais bem-sucedidos do setor florestal. Em 2004, o setor contava com 220 empresas localizadas em 16 estados e 450 municípios, gerando 100.000 empregos diretos e com pagamento de $\mathrm{R} \$ 2,2$ bilhões de impostos. Com um faturamento total de R \$ 23,1 bilhões em 2004, as exportações de celulose foram da ordem de 4,9 milhões de toneladas e o consumo aparente, de 4,95 milhões de toneladas. A produção brasileira de celulose vem crescendo desde 1950 e em 2004 alcançou 9,6 milhões de toneladas (BRACELPA, 2006).

O segmento brasileiro de celulose e papel tem todas as condições para continuar o crescimento acelerado e absorver cada vez mais,maior parcela de mercado, pois as empresas nacionais são competitivas devido

\footnotetext{
${ }^{1}$ Recebido em 26.07.2006 e aceito para publicação em 21.03.2007.

${ }^{2}$ Programa de Pós-Graduação em Ciência Florestal da UFV. E-mail:<naisysilva@ yahoo.com.br>.

${ }^{3}$ Departamento de Engenharia Florestal da UFV. E-mail: <marlosil@ufv.br>.

${ }^{4}$ Departamento de Economia Rural da UFV. E-mail: <jelima@ufv.br>.
} 
à alta produtividade dos reflorestamentos. Salientase que essa alta produtividade ocorre em razão das condições climáticas favoráveis à atividade florestal no país, possibilitando ciclos de rápido crescimento e de alta qualidade e ao baixo custo de produção em relação aos outros países.

Como observou Duerr (1960), entretanto as firmas do setor florestal devem-se preocupar mais com os fatores de produção, pois sobreviverão apenas as que trabalham com eficiência, isto é, as que trabalham com eficácia na utilização dos fatores de produção, medida pela relação entre produtividade e capacidade. Assim, torna-se de significativa importância determinar como os fatores de produção têm sido empregados na indústria de celulose no Brasil, para responder a uma série de questões na formulação de políticas de uso dos recursos.

Diante do exposto, cabe aqui mencionar que Bilas (1993) sugeriu, para descrever a relação entre nível de insumos utilizados na produção e a quantidade de produtos obtidos, o estudo da função de produção da firma.

É oportuno enfatizar que alguns estudos utilizando os fundamentos da teoria da produção já foram feitos na área florestal. Dentre eles, pode-se citar o de Ângelo et al. (2004), Lima Júnior et al. (1999), Rezende et al. (1996a,b).

Nesse contexto, os objetivos gerais deste trabalho foram especificar e ajustar a função de produção da indústria de celulose no Brasil, no ano de 2004. Mais especificamente, pretendeu-se quantificar a contribuição dos diferentes fatores de produção na indústria brasileira de celulose, calcular as produtividades média e marginal dos fatores e determinar como a indústria se apresenta com relação aos retornos à escala.

\section{MATERIAL E MÉTODO}

\subsection{Modelo teórico}

Tomaram-se como referência, para a realização deste estudo, os fundamentos da teoria da produção, indicados por Bilas (1993) e Silva (1996).

De acordo com Bilas (1993) e Silva (1996), se denominar por $\mathrm{X}_{\mathrm{i}}$ as quantidades dos fatores usados na produção da quantidade $\mathrm{Y}$ de produto, a função de produção pode ser representada conforme a equação 1 .

R. Árvore, Viçosa-MG, v.31, n.3, p.495-502, 2007
$Y=f\left(X_{1}, X_{2}, \ldots, X_{n}\right)$

$\mathrm{Na}$ forma Cobb-Douglas, a produção pode ser explicitada como sendo (equação 2):

$Y=\beta_{0} X_{1}^{\beta_{1}} X_{2}^{\beta_{2}} X_{3}^{\beta_{3}} \varepsilon$

em que:

$\mathrm{Y}=$ volume produzido (ou valor total da produção);

$X_{1}, X_{2}$ e $X_{3}=$ insumos de produção;

$\beta_{0}, \beta_{1}, \beta_{2}$ e $\beta_{3=}$ parâmetros a serem estimados; e

$\varepsilon=$ erro estocástico.

$\mathrm{O}$ produto físico médio de um fator $\mathrm{X}_{\mathrm{i}}\left(\mathrm{PFMe}_{\mathrm{xi}}\right)$ qualquer é positivo e decrescente à medida que se intensifica o uso do fator $\mathrm{X}_{\mathrm{i}}$. Mede a eficiência do fator variável e é definido como o quociente da divisão do volume produzido (ou valor total da produção) pela quantidade do fator utilizado (equação 3) (BILAS, 1993; SILVA, 1996).

$P F M e_{x i}=\frac{Y}{X}=\frac{A X_{1}^{\beta_{1}} X_{2}^{\beta_{2}} \ldots X_{n}^{\beta_{n}}}{X_{i}}=A X_{1}^{\beta_{1}} X_{2}^{\beta_{2}} \ldots X_{i}^{\beta_{i_{i}}-1} \ldots X_{n}^{\beta_{n}}>0$

O produto físico marginal de $\mathrm{X}_{\mathrm{i}}\left(\mathrm{PFMa}_{\mathrm{xi}}\right)$ é a variação na produção total que se origina da variação de uma unidade do fator de produção. É positivo se $\beta_{i}>0$ e decresce à medida que se intensifica o uso do fator $X_{i}$ (equação 4) (BILAS, 1993; SILVA, 1996).

$P F M a_{x i}=\frac{\partial Y}{\partial X_{i}}=\beta_{i} A X_{1}^{\beta_{i}} X_{2}^{\beta_{2}} \ldots X_{i}^{\beta-1} \ldots X_{n}^{\beta_{n}}=\beta_{i}\left(\frac{Y}{X_{i}}\right)>0$

A elasticidade parcial de produção de $\mathrm{X}_{\mathrm{i}}\left({ }_{\text {exi }}\right)$ é a relação entre a variação relativa da produção e a variação relativa do fator de produção (equação 5) (BILAS, 1993; SILVA, 1996).

$\varepsilon_{x_{i}}=\left[\beta_{i}\left(\frac{Y}{X_{i}}\right)\right]\left[\frac{X_{i}}{Y}\right]=\beta_{i}$

O retorno à escala é apresentado na equação 6 (BILAS, 1993; SILVA, 1996).

$G=\sum_{i=1}^{n} \beta_{i}$

Os seguintes casos são possíveis:

$\mathrm{G}>1=$ retornos crescentes à escala; 


$$
\begin{aligned}
& \mathrm{G}=1=\text { retornos constantes à escala; e } \\
& \mathrm{G}<1=\text { retornos decrescentes à escala. }
\end{aligned}
$$

A taxa marginal de substituição entre dois fatores $\mathrm{X}_{\mathrm{i}}$ e $\mathrm{X}_{\mathrm{j}}\left(\mathrm{TMS}_{\mathrm{xixj}}\right)$ é uma função linear da razão entre os insumos, com inclinação $\beta_{j} / \beta_{i}$. Refere-se à quantidade de um fator $X_{i}$ que a firma reduz pelo aumento na quantidade do fator $X_{\mathrm{j}}$, sem alterar a quantidade produzida (equação 7) (BILAS, 1993; SILVA, 1996).

$T M S_{x i x j}=\frac{\partial Y / \partial X_{j}}{\partial Y / \partial X_{i}}=\frac{P F M a_{x j}}{P F M a_{x i}}$

A partir das relações entre produto físico total (PFT), produto físico médio (PFMe) e produto físico marginal (PFMa), podem ser definidos três estágios de produção, como observou Bilas (1993).

No primeiro estágio de produção (I), o PFMe é sempre crescente, o PFMa é sempre maior que o PFMe e ambos são positivos. O PFT, por sua vez, apresentase crescente e a elasticidade de produção é maior que a unidade. Esse estágio é considerado um estágio irracional de produção, pois os insumos são alocados ineficientemente. Maior produtividade média poderia ser atingida pelo maior uso desse insumo. No terceiro estágio de produção (III), o PFT e o PFMe são decrescentes, o PFMa é negativo e a elasticidade de produção é menor que zero. Esse estágio também é considerado um estágio irracional de produção, uma vez que o emprego de unidades adicionais do insumo variável resultaria na redução do PFT, isto é, no crescimento do custo e sua redução da receita. $O$ segundo estágio de produção (II), por seu turno, é caracterizado por apresentar PFMe e PFMa decrescentes e positivos. Nesse estágio de produção, o PFMe é sempre superior ao PFMa, e a elasticidade de produção está entre 0 e 1. Esse é considerado o estágio racional de produção. O limite entre o primeiro e o segundo estágio ocorre onde o PFMe é máximo, isto é, quando o PFMe = PFMa. Nesse ponto, a elasticidade de produção é igual a 1 . Já o limite entre o segundo e o terceiro estágio acontece onde o PFT é máximo e o PFMa é nulo. Nesse ponto, a elasticidade de produção é zero (Figura 1) (BILAS, 1993; SILVA et al., 2002).

\subsection{Modelo empírico}

Para ajustar a função de produção da indústria de celulose no Brasil, foi proposto um modelo econométrico composto pela função de produção tipo Cobb-Douglas, adotando-se a forma log-log e o método dos Mínimos Quadrados Ordinários (MQO).
Assim, tem-se o modelo econométrico utilizado neste trabalho (equação 8).

$\log Q P=\beta_{0}+\beta_{1} \log T R+\beta_{2} \log M O+\beta_{3} \log C A+\varepsilon$

em que:

$\mathrm{QP}=$ quantidade produzida de celulose ao longo do ano de 2004, em toneladas;

$\mathrm{TR}=$ fator de produção terra, que corresponde à área com florestas das indústrias de celulose, em hectares;

$\mathrm{MO}=$ fator de produção trabalho, mensurado em equivalente homens, ou seja, número de trabalhadores na indústria de celulose;

$\mathrm{CA}=$ fator de produção capital, que representa a soma do valor das edificações e das máquinas e equipamentos, em $\mathrm{R} \$$;

$\log =\log$ aritmo;

$\beta_{0}, \beta_{1}, \beta_{2}$ e $\beta_{3}=$ parâmetros a serem estimados; e

$\varepsilon=$ erro estocástico.

Para testar a significância da regressão, utilizouse o teste F, de Snedecor. O grau de ajustamento da regressão foi avaliado por meio do coeficiente de determinação ajustado $\left(\mathrm{R}^{2}\right)$. Já a estatística "t", de Student, foi usada para testar a significância dos coeficientes individualmente.

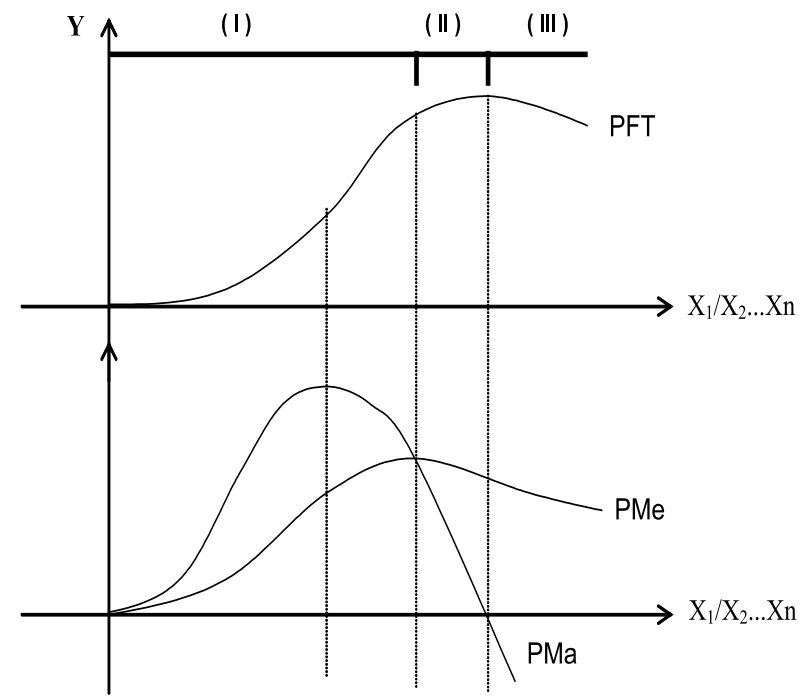

Fonte: Bilas (1993) e Silva et al. (2002).

Figura 1 - Os três estágios da função de produção clássica. Figure 1 - The three stages of the classic production function.

R. Árvore, Viçosa-MG, v.31, n.3, p.495-502, 2007 


\subsection{Fonte de dados}

Os dados utilizados são do ano de 2004. Não se trabalhou com um período mais atual porque alguns dados não estavam disponíveis.

A amostra foi composta por nove empresas, que em 2004 foram responsáveis por aproximadamente $90 \%$ da produção nacional de celulose.

A quantidade produzida de celulose por indústria, ao longo do ano de 2004, foi obtida conforme informações geradas por BRACELPA (2005).

O valor das máquinas e equipamentos e das edificações, assim como o número de trabalhadores e a área com florestas foi obtido no relatório anual de 2005 de cada empresa analisada, disponível nos seus sites. A relação das empresas em questão pode ser observada no Quadro 1.

\section{RESULTADOS E DISCUSSÃO}

Os resultados do ajuste da função de produção tipo Cobb-Douglas da indústria de celulose no Brasil encontram-se no Quadro 2.

O coeficiente de determinação ajustado $\left(\mathrm{R}^{2}\right)$ indica que $96,53 \%$ das variações ocorridas na produção de celulose no Brasil foram explicadas pelas variáveis predeterminadas no modelo. O coeficiente da variável terra foi significativo em nível de $2 \%$ de probabilidade e o das variáveis trabalho e capital, em nível de $1 \%$. O sinal positivo para coeficientes das variáveis terra, trabalho e capital indica que elas têm influência positiva na produção de celulose, e os valores no intervalo $[0,1)$ apontam que a produção ocorre no segundo estágio, para todos os fatores.

Do Quadro 2 extrai-se a equação que representa a função de produção, a qual é apresentada a seguir:

$Q P=3,29825 x T R^{0,468658} x M O^{0,461504} x C A^{0,253810}$

\section{Elasticidade parcial de produção}

Como o ajuste foi feito a partir de um modelo logarítmico, os parâmetros são interpretados como as elasticidades de produção.

A elasticidade de produção do fator terra foi 0,47 , evidenciando que um aumento de $10 \%$ em investimentos no fator terra ocasionaria aumento de $4,68 \%$ na produção de celulose ceteris paribus.

R. Árvore, Viçosa-MG, v.31, n.3, p.495-502, 2007
Quadro 1 - Relação das indústrias na amostra analisada Table 1 - List of the sampled industries

\begin{tabular}{lc}
\hline Empresas Analisadas & Referência usada \\
\hline Aracruz Celulose SA & $\mathrm{A}$ \\
Klabin SA & $\mathrm{B}$ \\
Votorantim Celulose e Papel SA & $\mathrm{C}$ \\
Suzano Bahia Sul & $\mathrm{D}$ \\
Celulose Nipo-Brasileira SA (Cenibra) & $\mathrm{E}$ \\
Ripasa SA Celulose e Papel & $\mathrm{F}$ \\
Veracel & $\mathrm{G}$ \\
Jari Celulose SA & $\mathrm{H}$ \\
Celulose Irani SA & $\mathrm{I}$ \\
\hline
\end{tabular}

Com relação ao fator de produção trabalho, podese dizer que aumento de $10 \%$ em investimento no trabalho acarretaria acréscimo de $4,61 \%$ na produção de celulose ceteris paribus. Já aumento de $10 \%$ em investimentos no fator capital contribuiria para o incremento de $2,53 \%$ na produção de celulose ceteris paribus.

Nota-se, assim, que os fatores terra e trabalho são os que contribuíram mais intensivamente na determinação da quantidade produzida de celulose. Em outras palavras, esses são os fatores com maior possibilidade de expandir a produção, pois apresentam as maiores elasticidades de produção.

Observou-se, também, que a indústria de celulose no Brasil está empregando os fatores terra, trabalho e capital racionalmente (II estágio de produção), haja vista que as elasticidades tanto da terra quanto do trabalho e do capital são maiores que zero e menores que a unidade. Então, sugere-se a manutenção dos fatores terra, trabalho e capital nos níveis atuais.

Ângelo et al. (2004) utilizaram a teoria da produção e a função tipo Cobb-Douglas para analisar a indústria de madeiras tropicais no pólo de Sinop (MT). Constaram que a referida indústria está empregando irracionalmente o fator mão-de-obra (III estágio de produção) e racionalmente o fator capital (II estágio de produção).

\section{Retorno à Escala}

O Quadro 3 apresenta o valor do coeficiente de escala. Pelo teste de Wald, verificou-se que se tem retorno constante à escala, em nível de $5 \%$ de probabilidade. Assim, em termos gerais, cada unidade adicional do fator variável, aplicado aos fatores fixos, aumenta a produção em iguais quantidades. 
Quadro 2 - Estimativas da função de produção de celulose Table 2 - Estimates for the wood pulp production function

\begin{tabular}{cccc}
\hline Variáveis Explicativas & Descrição & Coeficientes Estimados & Erro-Padrão \\
\hline Constante & & $0,518284^{\mathrm{ns}}$ & 0,930880 \\
Log TR & Terra & $0,468658^{*}$ & 0,149088 \\
Log MO & Trabalho & $0,461504^{* *}$ & 0,103548 \\
Log CA & Capital & $0,253810^{* *}$ & 0,067216 \\
\hline $\mathrm{R}^{2}=0,965347$ & & & $3,4567688^{*}$ \\
\hline
\end{tabular}

ns não-significativo, ${ }^{*}$ significativo a $2 \%$ e $* *$ significativo a $1 \%$.

Fonte: Resultados da pesquisa.

Quadro 3 - Retorno à escala para a indústria de celulose, Brasil, 2004

Table 3 -Return to scale for the wood pulp industry, Brazil, 2004

\begin{tabular}{cc}
\hline Fatores de Produção & Coeficientes Estimados \\
\hline TR & 0,468658 \\
MO & 0,461504 \\
CA & 0,253810 \\
\hline Retorno à escala & 1,183972 \\
\hline
\end{tabular}

Fonte: Resultados da Pesquisa.

Rezende et al. (1996b) também encontraram retorno à escala constante nas pequenas e grandes empresas florestais do Estado de Minas Gerais. Já Ângelo et al. (2004) verificaram que a indústria de madeiras tropicais no pólo de Sinop (MT) apresenta retornos decrescentes à escala.

\section{Produtividade Física Média}

No Quadros 4 são apresentados os resultados referentes às produtividades médias dos fatores de produção na indústria de celulose.

Constata-se, no Quadro 4, que se pode afirmar, quanto ao fator terra, que com 1 ha de florestas é possível produzir 4,5 toneladas de celulose.

Para o fator trabalho, pode-se aferir que a sua produtividade diária é de 0,63 tonelada de celulose, considerando 250 dias trabalhados/ano.

Quadro 4 - Produtividade física média dos fatores terra, trabalho e capital para a indústria de celulose, Brasil, 2004

Table 4-Average Physical Product of land, labor and capital factors for the wood pulp industry, Brazil, 2004

\begin{tabular}{cc}
\hline Fator de Produção & Produtividade Média \\
\hline TR & 4,5117 \\
MO & 157,7083 \\
CA & 0,7372 \\
\hline
\end{tabular}

Fonte: Resultados da Pesquisa.
O investimento médio de $\mathrm{R} \$ 1,00$ em capital produz 0,73 tonelada de celulose.

O Quadro 5 mostra a produtividade média dos fatores terra, trabalho e capital, por indústria no Brasil. Nesse quadro, verifica-se que a empresa $H$ é mais competitiva em relação aos fatores terra. No caso do fator trabalho, a empresa D é mais competitiva. Considerando-se o fator capital, a empresa $\mathrm{G}$ é mais competitiva.

\section{Produtividade Física Marginal}

No Quadro 6, apresenta-se o valor do produto marginal dos fatores de produção empregados na produção brasileira de celulose.

Quadro 5 - Produtividade média dos fatores terra, trabalho e capital, por indústria, Brasil, 2004

Table 5 - Average Physical Product of land, labor and capital factors, per industry, Brazil, 2004

\begin{tabular}{cccc}
\hline Empresas & PMe $(\mathrm{TR})$ & PMe $(\mathrm{MO})$ & PMe $(\mathrm{CA})$ \\
\hline A & 6,2425 & 243,9191 & 0,7241 \\
B & 6,0143 & 105,3871 & 3,1819 \\
C & 4,4918 & 266,1227 & 1,3071 \\
D & 5,2211 & 308,8759 & 0,3844 \\
E & 2,0702 & 98,3622 & 0,3772 \\
F & 5,4528 & 123,4063 & 0,5219 \\
G & 2,6997 & 120,0822 & 4,6615 \\
H & 21,8414 & 95,4690 & 3,5111 \\
I & 1,6239 & 45,9450 & 0,1807 \\
\hline
\end{tabular}

Fonte: Resultados da Pesquisa.

Quadro 6 - Produtividade marginal dos fatores terra, trabalho e capital para a indústria de celulose, Brasil, 2004

Table 6-Marginal product of land, labor and capital factors for wood pulp industry, Brazil, 2004

\begin{tabular}{cc}
\hline Fator de Produção & Produtividade Marginal \\
\hline TR & 2,1144 \\
MO & 72,7830 \\
CA & 0,1871 \\
\hline
\end{tabular}

Fonte: Resultados da Pesquisa.

R. Árvore, Viçosa-MG, v.31, n.3, p.495-502, 2007 
Os valores encontrados indicam que, ao empregar 1 ha a mais no fator terra, tem-se um acréscimo na produção da ordem de 2,11 toneladas ceteris paribus.

O acréscimo de uma unidade no fator trabalho acarretaria incremento na produção de celulose na ordem de grandeza de 72,78 toneladas ceteris paribus.

$\mathrm{O}$ aumento nos investimentos em bens de capital de $\mathrm{R} \$ 1,00$ resultaria em um incremento de 0,18 toneladas na produção de celulose ceteris paribus.

A produtividade marginal dos fatores de produção em cada empresa pode ser observada no Quadro 7, observando-se também que o PFMa, assim como o PFMe, indica que a empresa $\mathrm{H}$ é mais competitiva em relação ao fator de produção terra. A empresa D, no entanto, é mais competitiva em termos de fator trabalho. Considerandose o fator capital, a empresa $\mathrm{G}$ é mais competitiva.

O fato de o PFMa dos fatores ser positivo e menor que o PFMe também indica que as empresas estão operando racionalmente (II estágio de produção), ou seja, estão utilizando efetivamente os seus fatores produtivos.

\section{Taxa Marginal de Substituição}

A taxa marginal de substituição entre os fatores de produção, na indústria de celulose e em cada indústria, é apresentada nos Quadros 8 e 9, respectivamente.

Observa-se, por exemplo, que, quando se adiciona uma unidade de CA, devem ser abandonadas 388 unidades de MO para manter o mesmo nível de produção.

\section{Relação entre os fatores de produção}

A relação entre os fatores de produção, apresentada nos Quadros 10 e 11, demonstra o uso intensivo do fator capital na indústria de celulose no Brasil. O fator capital foi sempre superior em relação a outros fatores.
Quadro 7 - Produtividade marginal dos fatores terra, trabalho e capital, por indústria, Brasil, 2004

Table 7 - Marginal product of land, labor and capital factors per wood pulp industry, Brazil, 2004

\begin{tabular}{cccc}
\hline Empresas & PMa $\left(\mathrm{X}_{1}\right)$ & PMa $\left(\mathrm{X}_{2}\right)$ & PMa $\left(\mathrm{X}_{3}\right)$ \\
\hline A & 2,9255 & 112,5696 & 0,1838 \\
B & 2,8186 & 48,6366 & 0,8076 \\
C & 2,1051 & 122,8167 & 0,3318 \\
D & 2,4469 & 142,5475 & 0,0976 \\
E & 0,9702 & 45,3946 & 0,0958 \\
F & 2,5555 & 56,9525 & 0,1325 \\
G & 1,2652 & 55,4184 & 1,1831 \\
H & 10,2361 & 44,0593 & 0,8912 \\
I & 0,7611 & 21,2038 & 0,0459 \\
\hline
\end{tabular}

Fonte: Resultados da Pesquisa.

Quadro 8 - Taxa marginal de substituição entre os fatores de produção para a indústria de celulose, Brasil, 2004

Table 8 -Marginal rate of substitution between factors, for wood pulp industry, Brazil, 2004

\begin{tabular}{lc}
\hline Fatores & TMS \\
\hline MO/CA & 388,9648 \\
MO/TR & 34,4218 \\
TR/MO & 0,0291 \\
TR/CA & 11,3000 \\
CA/MO & 0,0026 \\
CA/TR & 0,0885 \\
\hline
\end{tabular}

Fonte: Resultados da Pesquisa.

Rezende et al. (1996a) também constataram que as empresas que atuam na região de Cerrado em Minas Gerais, mais especificamente no Alto São Francisco, são intensivas em capital.

Quadro 9 - Taxa marginal de substituição entre os fatores de cada indústria, Brasil, 2004 Table 9 -Marginal rate of substitution between factors for the wood pulp industry, Brazil, 2004

\begin{tabular}{cccccc}
\hline Empresas & TMS $_{\text {Mо CA }}$ & TMS $_{\text {MO TR }}$ & TMS $_{\text {TR MO }}$ & TMS $_{\text {TR CA }}$ & TMS $_{\text {CA MO }}$ \\
\hline A & 612,4716 & 38,4775 & 0,0260 & 15,9177 & 0,0016 \\
B & 60,2225 & 17,2553 & 0,0580 & 3,4901 & 0,0166 \\
C & 370,1946 & 58,3419 & 0,0171 & 6,3453 & 0,0028 \\
D & 1460,7312 & 58,2561 & 0,0172 & 25,0743 & 0,0007 \\
E & 474,0612 & 46,7863 & 0,0214 & 10,1325 & 0,0021 \\
F & 429,9129 & 22,2861 & 0,0449 & 19,2906 & 0,0023 \\
G & 46,8402 & 43,8008 & 0,0228 & 1,0694 & 0,0396 \\
H & 49,4407 & 4,3043 & 0,2323 & 11,4864 & 0,0518 \\
I & 462,2402 & 27,8598 & 0,0359 & 16,5917 & 0,9351 \\
\hline
\end{tabular}

Fonte: Resultados da Pesquisa.

R. Árvore, Viçosa-MG, v.31, n.3, p.495-502, 2007 
Quadro 10 - Relação entre os fatores terra, trabalho e capital e produto físico total, da indústria de celulose, Brasil, 2004

Table 10 - Ratio between land, labor and capital factors and total physical product, for wood pulp industry, Brazil, 2004

\begin{tabular}{cc}
\hline Relação & Resultados \\
\hline MO/TR & 0,0286 \\
CA/TR & 6,1197 \\
MO/CA & 0,0047 \\
PFT/TR & 4,5117 \\
PFT/MO & 157,7083 \\
PFT/CA & 0,7372 \\
\hline
\end{tabular}

Fonte: Resultados da Pesquisa.

Quadro 11 - Relação entre os fatores terra, trabalho e capital e produto físico total, de cada indústria, Brasil, 2004

Table 11 - Ratio between land, labor and capital factors and total physical product, per industry, Brazil, 2004

\begin{tabular}{lcccccc}
\hline Empresas & MO/TR & CA/TR & MO/CA & PFT/TR & PFT/MO & PFT/CA \\
\hline Aracruz & 0,0256 & 8,6205 & 0,0030 & 6,2425 & 243,9191 & 0,7241 \\
Klabin & 0,0571 & 1,8901 & 0,0302 & 6,0143 & 105,3872 & 3,1820 \\
VCP & 0,0169 & 3,4364 & 0,0049 & 4,4918 & 266,1228 & 1,3071 \\
Suzano & 0,0169 & 13,5794 & 0,0012 & 5,2211 & 308,8759 & 0,3845 \\
Cenibra & 0,0210 & 5,4874 & 0,0038 & 2,0703 & 98,3622 & 0,3773 \\
Ripasa & 0,0442 & 10,4472 & 0,0042 & 5,4528 & 123,4063 & 0,5219 \\
Veracel & 0,0225 & 0,5791 & 0,0388 & 2,6997 & 120,0823 & 4,6615 \\
Jari & 0,2288 & 6,2207 & 0,0368 & 21,8415 & 95,4691 & 3,5111 \\
Irani & 0,0353 & 8,9855 & 0,0039 & 1,6240 & 45,9450 & 0,1807 \\
\hline
\end{tabular}

Fonte: Resultados da Pesquisa.

\section{CONCLUSÃO}

Do presente trabalho é possível concluir que:

- A produção de celulose responde às variações na alocação dos fatores de produção, em uma ordem de grandeza em que $97,83 \%$ da produção é explicada pela alocação desses recursos. Isso indica que o modelo se ajustou bem aos dados.

- Os valores encontrados de elasticidade de produção, assim como os de PFMe e PFMa, indicam que os fatores de produção, sob análise, estão sendo empregados racionalmente na indústria de celulose no Brasil.

- A indústria de celulose no país apresenta retorno constante à escala.

- Os fatores terra e trabalho são os que mais contribuíram para a determinação da quantidade produzida de celulose no Brasil. São os que apresentam maior possibilidade de expandir a produção. Isso é favorável à indústria de celulose nacional, pois o país possui esses dois fatores em abundância.

- As empresas H, D e G, entre as firmas analisadas, são as mais competitivas em relação aos fatores terra, trabalho e capital, respectivamente.

- A indústria de celulose no país mostrou-se intensiva de capital, o que indica flexibilidade produtiva, atualização de produtos, redução de custos aumento da qualidade, e permite a competitividade da indústria de celulose nacional no mercado externo.

\section{AGRADECIMENTOS}

Os autores agradecem à Universidade Federal de Viçosa (UFV) pelo fornecimento da estrutura e pessoal; ao Conselho Nacional de Desenvolvimento Científico e Tecnológico (CNPq), pela concessão da bolsa de mestrado.

\section{REFERÊNCIAS}

ÂNGELO, H.; SILVA, G. F.; SILVA, V. S. M. Análise econômica da indústria de madeiras tropicais: o caso do pólo de Sinop, MT. Ciência Florestal, v.14, n.2, p.91-101, 2004.

\section{ASSOCIAÇÃO BRASILEIRA DOS FABICANTES DE CELULOSE E PAPEL - BRACELPA. \\ Relatório Anual, 2005.}

ASSOCIAÇÃO BRASILEIRA DOS FABRICANTES DE CELULOSE E PAPEL - BRACELPA. Disponível em:<http://www.bracelpa.org.br/bra/estatisticas/ index.html>. Acesso em: 20/01/2006.

BILAS, R. A. Teoria microeconômica. 12.ed. Rio de Janeiro: Forense Universitária, 1993. 404p.

DUERR, W.A. Fundamentos da economia florestal. Lisboa: Fundação Calouste Gulbenkian, 1960. 754p.

LIMA JÚNIOR, V. B.; REZENDE, J. L. P.; SILVA, M. L. Os estágios de produção e a idade ótima de corte: diferenças entre a teoria da produção instantânea e a preferência temporal na produção florestal. Revista Árvore, v.23, n.4, p.393-401, 1999.

R. Árvore, Viçosa-MG, v.31, n.3, p.495-502, 2007 
REZENDE, J. L. P.; SILVA, M. L.; TORRES, M. C.

Caracterização tecnológica da produção de madeira de eucalipto por meio do modelo Ulveling-Fletcher. Revista Árvore, v.20, n.2, p.207-215, 1996a.

REZENDE, J. L. P.; SILVA, M. L.; PEREIRA, J. J. Caracterização tecnológica da produção de pequenas e grandes empresas florestais do Estado de Minas Gerais. Revista Árvore, v.20, n.3, p.331-342, 1996b.
SILVA, L. A. C. A função de produção da agropecuária brasileira: diferenças regionais e evolução no período 1975 1985. 1996. 157f. Tese (Doutorado em Ciências) Escola Superior de Agricultura Luiz de Queiroz, Piracicaba, 1996.

SILVA, M. L.; JACOVINE, L.A. G; VALVERDE, S. R. Economia florestal. Viçosa, MG: Universidade Federal de Viçosa, 2002. 178p. 